${ }^{2}$ Clinic of General, Gastrointestinal, Colorectal and Oncological Surgery, Faculty of Medicine, Ludwik Rydygier Collegium Medicum in Bydgoszcz, Nicolaus Copernicus University in Toruń, Poland

${ }^{3}$ Department of Vascular and Internal Diseases, Faculty of Health Sciences, Ludwik Rydygier Collegium Medicum in Bydgoszcz, Nicolaus Copernicus University in Toruń, Poland

\title{
Selected nutritional risk parameters in patients with laryngeal cancer - a comparison with other patients hospitalized in a Department of Laryngology and patients with colorectal cancer
}

\author{
Corresponding author: \\ Jacek Budzyński \\ Department of Vascular and Internal \\ Diseases, Faculty of Health Sciences, \\ Ludwik Rydygier Collegium Medicum \\ in Bydgoszcz, Nicolaus Copernicus \\ University in Toruń, Poland \\ e-mail: budz@cps.pl
}

Medical Research Journal 2018 ; Volume 3, Number 4, 188-194 10.5603/MRJ.a2018.0031 Copyright (C) 2018 Via Medica ISSN 2451-2591

\begin{abstract}
Background: It is assumed that neoplasm greater in size may affect a patients' nutritional status and prognosis stronger than smaller one. The aim of this study was to compare the nutritional status and prognosis of patients with laryngeal cancer (LC), recognized as tumour smaller in size, and patients with colorectal cancer (CRC) who were hospitalized in our hospital during the one year period.

Methods: The retrospective review of medical documentation of all 1,134 patients hospitalized in a Department of Otolaryngology.

Results: The laryngeal tumour was smaller than colorectal. Nutritional risk concerned $9 \%$ of patients with LC, was greater than in patients with other laryngeal disorders (1.4\%), and lower than in patients with CRC (37\%). A Nutritional Risk Screening (NRS) 2002 score $\geq 3$ was the only significant factor influencing the risk of in-hospital all-cause mortality, 14- and 30-day readmissions in patients with LC, and the risk of 14-day rehospitalization in patients with CRC

Conclusions: Risk of malnutrition in patients with LC was lower than in counterparts with CRC, and concern $9 \%$ and $37 \%$ of patients, respectively. Nutritional risk diagnosed in patients with LC had a stronger association with the prevalence of the measured outcomes (in-hospital death, the risk of 14-day and 30-day readmission, length of hospitalization) than in individuals with CRC.

Key words: nutritional status; laryngeal cancer; colorectal cancer
\end{abstract}

Med Res J 2018; 3 (4): 188-194

\section{Introduction}

Malnutrition and cachexia are common disorders in patients with cancer. Among individuals with neoplasms of the head or larynx, these disorders affect about $35-50 \%$ of patients [1]. Malnutrition is an important risk factor for in-hospital mortality, rehospitalization, prolonged general inpatient hospital stays [2], as well as the incidence of complications [1, 3, 4]. In patients treated for laryngeal cancer (LC), these complications include immunodeficiencies, poor wound healing, wound infection, anastomotic leakage, fistula, respiratory insufficiency, and sepsis. The many nutritional risk parameters (e.g. blood concentrations of albumin, prealbumin, transferrin, as well as lymphocyte count) $[5,6]$, have been analyzed as factors affecting the outcomes of LC treatment, but even low body mass index (BMI) before surgery was related to poor prognosis in patients with squamous laryngeal cancer [7] and individuals with colorectal cancer (CRC) [8]. The causes of malnutrition in these patients were not only associated with cancer-related cachexia, but also to local tumour effects (e.g. pharyngeal dysphagia, odynophagia and ileus), anorexia, and alcoholism [1]. These factors may be responsible for greater nutritional risk among patients with head and neck cancers compared to neo- 
plasms in other localizations. To check this hypothesis, we analyzed the values of some nutritional parameters over a one-year period in patients with LC and CRC, hospitalized, respectively, in the Departments of Otolaryngology and Surgery at our hospital, and compared the nutritional risk.

\section{Patients and methods}

We performed an analysis of the medical documentation of 1,134 patients treated in the Department of Otolaryngology and 92 patients with CRC treated in the Department of Surgery between July $1^{\text {st }}, 2014$ and June $30^{\text {th }}, 2015$ in a university hospital. The premise for choosing CRC as the counterpart for patients with LC was that both neoplasms can affect patients' nutritional status through changes in the functioning of the alimentary tract.

The following clinical data, nutritional screening scores and nutritional assessment parameters were evaluated: age, gender, number of days hospitalized, hospitalization mode (whether urgent or scheduled), in-hospital all-cause mortality, non-elective readmission, Nutritional Risk Screening (NRS) 2002 score (a score of at least 3 points for the questionnaire indicates a risk of malnutrition), body mass, height, BMI, blood concentration of hemoglobin, total cholesterol, low-density lipoprotein (LDL) cholesterol, triglycerides, glucose, albumin, C-reactive protein (CRP), and absolute lymphocyte count. All the biochemical parameters included in the analysis were the first determinations during the respective hospitalizations. The following secondary parameters were also calculated:

- an "ideal weight" was calculated according to the Lorentz formula: for female patients, ideal weight $=$ [height $(\mathrm{cm})-100]-\{$ [height $(\mathrm{cm})$ $-150] / 2\}$; and for male patients, ideal weight $=$ [height (cm) - 100] $-\{$ [height (cm) - 150]/4\} [9];

- an "absolute difference between the actual and ideal body weight" was calculated according to the following formula: actual body mass — ideal weight;

- a "relative difference between actual and ideal body weight" was calculated according to the following formula: 100 x (actual body mass - ideal weight)/ actual body mass;

- body mass deficit was defined as a negative value of the "absolute difference between the actual and ideal body weight";

- the Nutritional Risk Index (NRI) was calculated according to the following formula: $\mathrm{NRI}=1.519 \mathrm{x}$ blood albumin concentration $(\mathrm{g} / \mathrm{l})+41.7 \times$ actual body mass/ideal body mass [9,10];

- Onodera's (Preoperative) Prognostic Nutritional Index (OPNI) was calculated as $10 \times$ serum albumin concentration $(\mathrm{g} / \mathrm{dl})+0.005 \times$ lymphocyte count $\left(\right.$ per $\left.\mathrm{mm}^{3}\right)$ [11-15];
- blood CRP/albumin ratio.

The above acted as nutritional screening scores and nutritional assessment parameters [2].

The diagnosis of cancerous and non-cancerous disease in the respective patients was determined according to the ICD-10 Classification of Diseases. The LC and CRC staging was assessed according to the $7^{\text {th }}$ edition of the Cancer Staging Manual of the American Joint Committee on Cancer [16].

\section{Measured outcomes}

The following outcomes were measured: hospital length of stay (LOS; the number of days hospitalized), in-hospital all-cause mortality, and non-elective readmission (the second and subsequent hospitalizations during the period analyzed) in the 14-day and 30-day periods following discharge.

\section{Bioethics}

The investigation was conducted in compliance with the Declaration of Helsinki for medical research.

\section{Statistics}

Statistical analysis was conducted using licensed versions of STATISTICA statistical software (a data analysis software system), StatSoft, Inc. (2017), version 13.1. The normal distribution of the study variables was checked using the Shapiro-Wilk test. The results were mainly presented as the mean \pm standard deviation (SD), or $\mathrm{n}, \%$. The statistical significance of differences between groups was verified using the Student's t-test and $\mathrm{Chi}^{2}$ test. Spearman rank correlation was also used. The statistical significance level was set at a $p$-value $<0.05$. The odds ratio (OR) was defined as the odds that an outcome will occur with the association of some value of an estimated variable (a clinical or biochemical parameter), compared to the odds of the outcome occurring in the absence of that association. The OR was calculated according to the following formula: the product of the number of subjects with the measured outcome and the presence of the variables analyzed (exposed cases) and the number of subjects without the presence of the variables analyzed (unexposed non-cases) divided by the product of the numbers of exposed non-cases and unexposed cases. For this purpose, we used free statistical calculators (e.g. https://www. medcalc.org/calc/odds_ratio.php). These analyses were performed both per hospitalization and per patient. 
Table 1. Demographic and clinical data of the patients analyzed

\begin{tabular}{|c|c|c|c|}
\hline Feature & $\begin{array}{l}\text { Patients with } \\
\text { laryngeal cancer } \\
(n=33)\end{array}$ & $\begin{array}{l}\text { Patients in the Department } \\
\text { of Laryngology without } \\
\text { a diagnosis of laryngeal } \\
\text { cancer }(n=1,101)\end{array}$ & $\begin{array}{l}\text { Patients with } \\
\text { colorectal cancer } \\
\quad(n=92)\end{array}$ \\
\hline Age (years) & $62.0 \pm 10.9$ & $47.3 \pm 18.6+$ & $69.3 \pm 11.3+$ \\
\hline Age $\geq 65$ years $(n, \%)$ & $13(39.4 \%)$ & $201(18.3 \%)+$ & $82(89.1 \%)+$ \\
\hline Male gender (n, \%) & $31(93.9 \%)$ & $602(54.7 \%)+$ & $65(70.7 \%)+$ \\
\hline Tumor size $(\mathrm{cm})$ & $2.74 \pm 1.02$ & & $4.52 \pm 2.69+$ \\
\hline \multicolumn{4}{|l|}{ Neoplastic disease stage } \\
\hline I (n, \%) & $9(27.3 \%)$ & & $28(30.4 \%)$ \\
\hline II $(\mathrm{n}, \%)$ & $5(15.1 \%)$ & & $20(21.7 \%)$ \\
\hline III (n, \%) & $9(27.3 \%)$ & & $21(22.8 \%)$ \\
\hline IV (n, \%) & $10(30.3 \%)$ & & $23(25.0 \%)$ \\
\hline Diabetes (n, \%) & $3(9.1 \%)$ & $37(3.4 \%)$ & $20(21.7 \%)$ * \\
\hline Duration of hospitalization (days) & $9.6 \pm 6.8$ & $3.1 \pm 2.9+$ & $10.7 \pm 9.5$ \\
\hline In-hospital death (n, \%) & $1(3 \%)$ & $2(0.18 \%)$ * & $9(9.8 \%)+$ \\
\hline Rehospitalization within 14 days (n, \%) & $1(3 \%)$ & $5(0.45 \%)$ & $8(8.7 \%)+$ \\
\hline Rehospitalization within 30 days (n, \%) & $1(3 \%)$ & $13(1.2 \%)$ & $13(14.1 \%)$ \\
\hline Body mass (kg) & $77.7 \pm 12.3$ & $75.5 \pm 17.4$ & $75.90 \pm 17.4$ \\
\hline Height $(\mathrm{cm})$ & $176.9 \pm 8.2$ & $170.2 \pm 11.6$ * & $167.1 \pm 8.9+$ \\
\hline BMI $\left(\mathrm{kg} / \mathrm{m}^{2}\right)$ & $24.9 \pm 4.1$ & $25.9 \pm 4.9$ & $27.1 \pm 5.4$ * \\
\hline Ideal body weight (kg) & $69.7 \pm 6.7$ & $63.71 \pm 9.1+$ & $61.7 \pm 7.4+$ \\
\hline $\begin{array}{l}\text { Absolute difference between actual and ideal } \\
\text { body weight }(\mathrm{kg})\end{array}$ & $8.05 \pm 12.3$ & $11.81 \pm 13.8$ & $14.3 \pm 15.3$ * \\
\hline $\begin{array}{l}\text { Relative difference between actual and ideal body } \\
\text { weight (\%) }\end{array}$ & $10.3 \pm 14.8$ & $12.5 \pm 17.4$ & $15.8 \pm 15.6$ * \\
\hline Body mass deficit (n, \%) & $8(24.2 \%)$ & $192(17.4 \%)$ & $17(18.5 \%)$ \\
\hline NRS-2002 score & $1.0 \pm 1.2$ & $0.2 \pm 0.6+$ & $2.14 \pm 1.5+$ \\
\hline NRS-2002 $\geq 3$ score & $3(9.1 \%)$ & $15(1.4 \%)$ * & $34(37.0 \%)+$ \\
\hline NRI & $50.1 \pm 9.3$ & $54.9 \pm 13.5$ & $54.3 \pm 11.8$ \\
\hline Albumin (g/l) & $3.2 \pm 0.6$ & $3.3 \pm 0.8$ & $3.1 \pm 0.8$ \\
\hline $\mathrm{CRP}(\mathrm{mg} / \mathrm{dl})$ & $49.4 \pm 53.2$ & $64.2 \pm 117.9$ & $78.8 \pm 75.4$ \\
\hline CRP/albumin ratio & $19.84 \pm 20.9$ & $54.14 \pm 99.5$ & $24.6 \pm 31.2$ \\
\hline Blood lymphocyte count (G/l) & $1.7 \pm 0.7$ & $2.0 \pm 1.0$ & $1.8 \pm 2.9$ \\
\hline OPNI & $31.8 \pm 5.6$ & $31.2 \pm 9.3$ & $30.9 \pm 7.9$ \\
\hline Hemoglobin (g/dl) & $13.5 \pm 2.2$ & $14.0 \pm 1.8$ & $11.3 \pm 2.5$ \\
\hline Cholesterol (mg/dl) & $179.1 \pm 49.3$ & $190.9 \pm 67.0$ & $150.0 \pm 60.9$ \\
\hline LDL cholesterol (mg/dl) & $76.7 \pm 10.0$ & $128.3 \pm 59.8$ & $105.7 \pm 43.7$ \\
\hline Triglycerides (mg/dl) & $120.7 \pm 40.7$ & $126.2 \pm 74.5$ & $123.4 \pm 67.5$ \\
\hline Glucose (mg/dl) & $118.2 \pm 36.4$ & $108.6 \pm 44.7$ & $131.0 \pm 50.1$ \\
\hline
\end{tabular}

BMI — body mass index; NRS — Nutritional Risk Screening; NRI — Nutritional Risk Index; CRP — C-reactive protein; OPNI — Onodera's Prognostic Nutritional Index; LDL - low-density lipoprotein. Student's t-test in relation to patients with laryngeal cancer: ${ }^{\star}-p<0.05 ;+-p<0.001$

\section{Results}

Patients with LC were predominantly male and younger than patients with CRC but, on average, older than other patients admitted to the Department of
Otolaryngology (Tab.1). The tumour size in patients with LC was lower than in individuals with CRC. The percentages of patients with the respective clinical stage of neoplasm were similar (Tab.1). The duration of hospitalization of patients with LC was similar to 
that of patients with CRC. In relation to nutritional risk, compared to patients with CRC, patients with LC had a significantly lower BMl value, and both a lower absolute and relative difference between actual and ideal body weight (Tab.1). They also had lower nutritional risk expressed by NRS-2002 score and a lower prevalence of an NRS-2002 score $\geq 3$. However, the remaining biochemical parameters of nutritional status did not differ in patients with LC compared with those with CRC.

In patients with LC, the number of hospitalization days correlated significantly and positively with NRS2002 score $(R=0.47 ; p=0.006)$; however, in patients with $\mathrm{CRC}$, the length of hospital stay was significantly associated with patients' age $(R=0.21 ; p=0.022)$, hyperglycemia (blood concentration of triglycerides greater than $200 \mathrm{mg} / \mathrm{dl}$ ) on admission $(R=0.34$; $p=0.016)$, NRS-2002 score $(R=0.39 ; p=0.0001)$, and body weight deficit $(R=0.21 ; p=0.039)$. LC stage correlated significantly with an NRS-2002 score $\geq 3$. It was also related to the length of patients' in-hospital stay. CRC stage significantly positively correlated with blood lymphocyte count, and negatively with BMI, NRI and relative difference between actual and ideal body weight (Tab.2).

Next, we analyzed factors influencing the occurrence of the measured outcomes (Tab.3). We found that only an NRS-2002 score $\geq 3$ had a significant effect on the risk of in-hospital all-cause mortality, 14- and 30-day readmissions in patients with LC, and the risk of 14-day rehospitalization in patients with $\mathrm{CRC}$.

\section{Discussion}

The main rationale for this study was to check what is more important for patients nutritional risk, tumour size or its localization. To test this hypothesis we compared nutritional risk and prevalence of measured outcomes, related to nutritional risk among patients with LC and CRC. Moreover, in order to better evaluation of the clinical importance of disease localization, we compared some clinical data between patients with LC and the other laryngeal disorders required hospitalization. This study, to the best of our knowledge, is the first comparison of parameters of nutritional screening scores and nutritional status assessment parameters between patients with $L C$ and CRC, the sixth and the second most common cancers in the world, respectively [17]. We found that patients with LC compared to individuals with CRC had significantly lower BMI values and a non-significantly lower prevalence of body mass deficit (Tab. 1). At the same time, they had a lower nutritional risk, expressed as an NRS-2002 score, and a lower absolute and relative difference between actual and ideal body weight (Tab. 1). Only $9 \%$ of our patients with LC had increased nutritional risk expressed as a score of at least

Table 2. Spearman's correlations of laryngeal cancer (LC) and colorectal cancer (CRC) clinical stage with selected parameters of nutritional screening and assessment

\begin{tabular}{lcccc}
\hline Cancer stage correlation with & \multicolumn{2}{c}{ LC (n= 33) } & \multicolumn{2}{c}{ CRC (n= 92) } \\
\cline { 2 - 5 } & $\mathbf{R}$ & $\mathbf{p}<$ & $\mathbf{R}$ & $\mathbf{p}$ \\
\hline Age & -0.17 & 0.33 & -0.02 & 0.84 \\
Gender (female/male) & -0.02 & 0.95 & -0.1 & 0.36 \\
In-hospital length of stay (days) & 0.59 & 0.001 & 0.05 & 0.63 \\
BMI (kg/m ${ }^{2}$ ) & 0.10 & 0.64 & -0.23 & 0.039 \\
NRS-2002 score & 0.32 & 0.08 & 0.23 & 0.07 \\
NRS-2002 score $\geq 3$ & 0.40 & 0.024 & 0.23 & 0.07 \\
Relative difference between actual and ideal body weight (\%) & 0.02 & 0.91 & -0.23 & 0.044 \\
NRI & 0.71 & 0.02 & -0.45 & 0.035 \\
Hemoglobin (g/dl) & -0.28 & 0.23 & -0.17 & 0.11 \\
Total blood cholesterol (mg/dl) & -0.04 & 0.95 & 0.05 & 0.88 \\
Blood glucose (mg/dl) & -0.13 & 0.65 & 0.25 & 0.15 \\
CRP (mg/dl) & 0.37 & 0.47 & 0.12 & 0.46 \\
Lymphocyte count (G/l) & 0.14 & 0.77 & 0.44 & 0.019 \\
Blood albumin (g/l) & -0.35 & 0.056 & 0.10 & 0.63 \\
CRP/albumin ratio & 0.35 & 0.055 & 0.22 & 0.23 \\
OPNI & -0.35 & 0.056 & -0.06 & 0.79 \\
\hline BMI- body mas
\end{tabular}

BMI — body mass index; NRS — Nutritional Risk Screening; NRI — Nutritional Risk Index; CRP — C-reactive protein; OPNI — Onodera's (Preoperative) Prognostic Nutritional Index (OPNI) 
3 in the NRS-2002 questionnaire compared to $37 \%$ of individuals with CRC (Tab. 1). Other authors have shown that malnutrition prevalence among patients with head and neck cancers was greater than in our investigation and amounted to $30-50 \%[1,6]$. Kwag et al. reported a prevalence of malnutrition in patients with CRC in the Korean population at a level similar to ours [18]. However, other studies have shown that the prevalence of malnutrition among patients with CRC amounted to between $19.2 \%$ [19] and 30-60\% [20]. Until now, only a few authors have compared the nutritional status of patients with neoplasms in different localizations. In a study by Du et al. [21], the proportions of patients with low blood levels of albumin, prealbumin, transferrin, red blood cells, hemoglobin and hematocrit were higher for gastric cancer than for colon cancer, which was explained by the greater susceptibility of gastric patients to malnutrition and loss of fatty tissue.

It is also known that hypoalbuminemia significantly increases the length of hospital stay, rates of surgical site infections, and the risk of enterocutaneous fistula formation and deep vein thrombosis, particularly in patients with CRC $[22,23]$. Moreover, serum albumin was superior to prealbumin for predicting short-term recurrence in patients with operable CRC [24], and the morbidity and mortality rates in patients with CRC decreased by $7.3 \%$ and $15.6 \%$, respectively, for each $0.1 \mathrm{~g} / \mathrm{dl}$ increase in preoperative serum albumin level. ${ }^{23}$ However, in our study, blood albumin concentration was similar both in patients with LC and CRC, as well as in the patients in the Department of Laryngology without a diagnosis of laryngeal cancer (Tab. 1). In our patients with LC, blood albumin concentration correlated with neoplasm stage only with borderline statistical significance, similarly to derivative composed parameters, such as the CRP/albumin ratio and OPNI (Tab. 2). The last parameter, OPNI, which is the product of blood albumin concentration and lymphocyte count [11-15], linked LC with CRC because, in our patients with $\mathrm{CRC}$, neoplasm advancement was associated with lymphocyte count, NRI and BMI (Tab. 2).

It is known that a patient's nutritional status, particularly malnutrition but also obesity, are common but poor prognosis factors in individuals with cancers in a number of localizations $[1,6,20]$. We also found that nutritional risk assessed using the NRS-2002 survey was related to an increased prevalence of the measured outcomes, such as patients' in-hospital mortality and readmissions (Tab. 3). This demonstrates the necessity for further studies focused on the clinical importance of nutritional status assessment in patients with $L C$, as well as the need to evaluate the effectiveness of nutritional support and its financial impact on health services [6]. On the other hand, it should be underlined that, other than the NRS-2002, we did not find any single parameter of nutritional status assessment which related both to LC and CRC clinical stage (Tab. 2) and could be used to predict patients' outcomes (Tab. 3). In our review of the literature, we did not find one recommended nutritional screening scores or nutritional assessment parameters dedicated to patients with LC either [5-7]; however, for patients with $\mathrm{CRC}$, the most frequently used instruments for this purpose were as follows: the NRS-2002 [18-19], Malnutrition Universal Screening Tool (MUST), Subjective Global Assessment (SGA) [25, 26], Patient-Generated Subjective Global Assessment (PG-SGA), NRI, and OPNI [11-15, 25, 26]. In the study by Kwag et al. [18], the NRS-2002 was an independent predictor of postoperative complications (OR 3.05; $p=0.045)$, such as anastomotic leakage and wound infection in patients with CRC.

The practical importance of our observations is that both in patients with LC and CRC, nutritional screening and assessment should be performed using more than one diagnostic tool at the same time. This approach to patient management might potentially help identify patients with an increased risk of malnutrition and postoperative complications. An increased NRS-2002 score may also identify those LC and CRC patients who might potentially benefit from nutritional support [27-30].

As with most authors, we could not avoid some methodological shortcomings that could have influenced the strength of the deductions based on our results. The main limitation is a retrospective study design based on documentation analysis, although such a study design was described previously [4]. Moreover, our sample size was small, and we observed a low number of measured outcomes. It should also be taken into consideration that the clinical outcomes analyzed might be influenced by a number of factors other than nutritional status alone, e.g. main disease and comorbidity severity, which may also bias the results obtained. Such an observation is also justified by the analysis of Table 1, which shows an imbalance in potential confounding factors between groups, mainly concerning age, gender, and diabetes prevalence.

\section{Conclusions}

The average nutritional risk in patients with laryngeal cancer amounted to $9 \%$ and was greater than in individuals with other conditions that required hospitalization in the Department of Otolaryngology but lower than in patients with CRC (37\%). Nutritional risk diagnosed in patients with laryngeal cancer had a stronger association with the prevalence of the measured outcomes (in-hospital death, the risk of 14-day and 30-day readmission, length of hospitalization) than in individuals with colorectal cancer. However, due to 


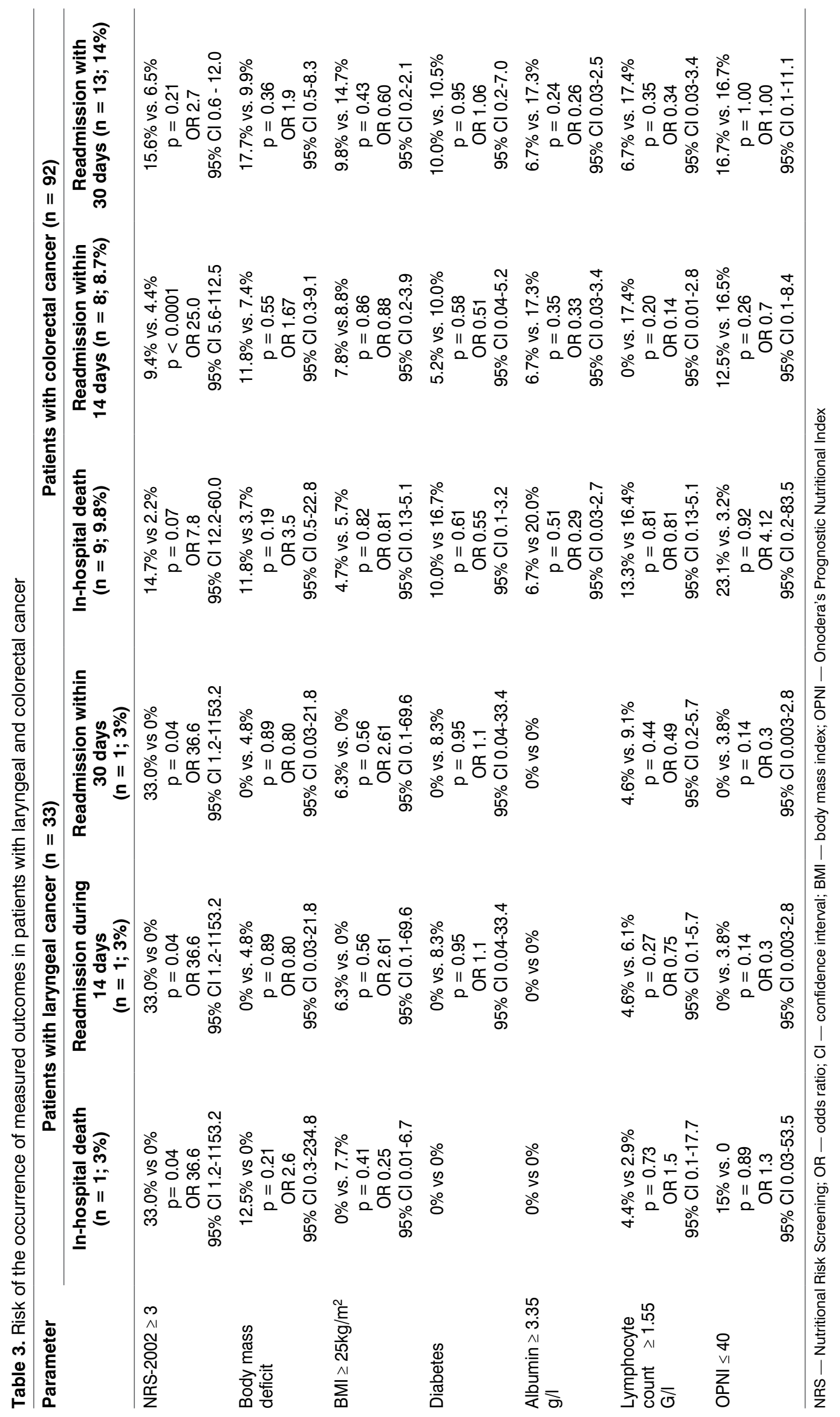


study limitations, the clinical significance of nutritional risk assessment in patients with LC and its financial impact on health services need further investigation.

\section{References}

1. Casas-Rodera P, Gómez-Candela C, Benítez S, et al. Immunoenhanced enteral nutrition formulas in head and neck cancer surgery: a prospective, randomized clinical trial. Nutr Hosp. 2008; 23(2): 105-110, indexed in Pubmed: 18449445

2. Budzyński J, Tojek K, Czerniak B, et al. Scores of nutritional risk and parameters of nutritional status assessment as predictors of in-hospital mortality and readmissions in the general hospital population. Clin Nutr. 2016; 35(6): 1464-1471, doi: 10.1016/j.clnu.2016.03.025, indexed in Pubmed: 27113120

3. Bruzgielewicz A, Hamera M, Osuch-Wójcikiewicz E. [Nutritional status of patients with cancer of larynx and hypopharyx]. Otolaryngol Pol. 2009; 63(2): 141-146, doi: 10.1016/S0030-6657(09)70095-2, indexed in Pubmed: 19681485

4. Collins MM, Wight RG, Partridge G. Nutritional consequences of radiotherapy in early laryngeal carcinoma. Ann R Coll Surg Engl. 1999; 81(6): 376-381, indexed in Pubmed: 10655889

5. De Lu, Izaola O, Aller R. Nutritional status in head and neck cance patients. Eur Rev Med Pharmacol Sci. 2007; 11: 239-43.

6. De Luis DA, Izaola O, Terroba MC, et al. Effect of three different doses of arginine enhanced enteral nutrition on nutritional status and outcomes in well nourished postsurgical cancer patients: a randomized single blinded prospective trial. Eur Rev Med Pharmacol Sci. 2015; 19(6): 950-955, indexed in Pubmed: 25855918.

7. Li ZQ, Zou L, Liu TR, et al. Prognostic value of body mass index before treatment for laryngeal squamous cell carcinoma. Cancer Biol Med. 2015; 12(4): 394-400, doi: 10.7497/j.issn.2095-3941.2015.0043, indexed in Pubmed: 26779376.

8. Kroenke $\mathrm{CH}$, Neugebauer $\mathrm{R}$, Meyerhardt J, et al. Analysis of Body Mass Index and Mortality in Patients With Colorectal Cancer Using Causa Diagrams. JAMA Oncol. 2016; 2(9): 1137-1145, doi: 10.1001/jamaoncol.2016.0732, indexed in Pubmed: 27196302.

9. Bouillanne O, Morineau G, Dupont C, et al. Geriatric Nutritional Risk Index: a new index for evaluating at-risk elderly medical patients. Am J Clin Nutr. 2005; 82(4): 777-783, doi: 10.1093/ajcn/82.4.777, indexed in Pubmed: 16210706

10. Abd-El-Gawad WM, Abou-Hashem RM, El Maraghy MO, et al. The validity of Geriatric Nutrition Risk Index: simple tool for prediction of nutritional-related complication of hospitalized elderly patients. Comparison with Mini Nutritional Assessment. Clin Nutr. 2014; 33(6): 1108-1116 doi: 10.1016/j.clnu.2013.12.005, indexed in Pubmed: 24418116

11. Shibutani M, Maeda $\mathrm{K}$, Nagahara $\mathrm{H}$, et al. The prognostic significance of the postoperative prognostic nutritional index in patients with colorectal cancer. BMC Cancer. 2015; 15: 521, doi: 10.1186/s12885015-1537-x, indexed in Pubmed: 26177820.

12. Jian-Hui C, Iskandar EA, Cai SI, et al. Significance of Onodera's prognostic nutritional index in patients with colorectal cancer: a large cohort study in a single Chinese institution. Tumour Biol. 2016; 37(3) 3277-3283, doi: 10.1007/s13277-015-4008-8, indexed in Pubmed: 26438061

13. Ikeya T, Shibutani M, Maeda K, et al. Maintenance of the nutritional prognostic index predicts survival in patients with unresectable metastatic colorectal cancer. J Cancer Res Clin Oncol. 2015; 141(2): 307-313 doi: 10.1007/s00432-014-1799-8, indexed in Pubmed: 25124497.

14. Maeda K, Shibutani M Otani $\mathrm{H}$, et al. Low nutritional prognostic index correlates with poor survival in patients with stage IV colorectal can- cer following palliative resection of the primary tumor. World J Surg. 2014: 38(5): 1217-1222, doi: 10.1007/s00268-013-2386-x, indexed in Pubmed: 24305937

15. Nozoe $\mathrm{T}$, Kohno M, Iguchi $\mathrm{T}$, et al. The prognostic nutritional index can be a prognostic indicator in colorectal carcinoma. Surg Today. 2012; 42(6): 532-535, doi: 10.1007/s00595-011-0061-0, indexed in Pubmed: 22139087

16. Edge SB, Byrd DR, Compton CC, et al. The AJCC cancer staging manual. The 7th edition. Springer, London. ; 2010.

17. Pai PC, Chuang CC, Tseng CK, et al. Impact of pretreatment body mass index on patients with head-and-neck cancer treated with radiation. Int J Radiat Oncol Biol Phys. 2012; 83(1): e93-e9e100, doi: 10.1016/j. ijrobp.2011.11.071, indexed in Pubmed: 22342298.

18. Kwag SJ, Kim JG Kang WK et al. The nutritional risk is a independent factor for postoperative morbidity in surgery for colorectal cancer. Ann Surg Treat Res. 2014; 86(4): 206-211, doi: 10.4174/astr.2014.86.4.206, indexed in Pubmed: 24783180.

19. Jia ZY, Yang J, Tong DN, et al. Screening of nutritional risk and nutritional support in general surgery patients: a survey from Shanghai, China. Int Surg. 2015; 100(5): 841-848, doi: 10.9738/INTSURG-D-14-00245.1, indexed in Pubmed: 26011204.

20. Lopes JP, de Castro Cardoso Pereira PM, dos Reis Baltazar Vicente AF, et al. Nutritional status assessment in colorectal cancer patients. Nutr Hosp. 2013; 28(2): 412-418, doi: 10.3305/nh.2013.28.2.6173, indexed in Pubmed: 23822693

21. Du Yp, Li LI, He Q, et al. [Nutritional risk screening and nutrition assessment for gastrointestinal cancer patients]. Zhonghua Wei Chang Wai Ke Za Zhi. 2012; 15(5): 460-463, indexed in Pubmed: 22648839.

22. Truong A, Hanna MH, Moghadamyeghaneh Z, et al. Implications of preoperative hypoalbuminemia in colorectal surgery. World J Gastrointest Surg. 2016; 8(5): 353-362, doi: 10.4240/wjgs.v8.i5.353, indexed in Pubmed: 27231513

23. Chiang JM, Chang CJ, Jiang SF, et al. Pre-operative serum albumin level substantially predicts post-operative morbidity and mortality among patients with colorectal cancer who undergo elective colectomy. Eur J Cancer Care (Engl). 2017; 26(2), doi: 10.1111/ecc.12403, indexed in Pubmed: 26526411

24. Fujii $\mathrm{T}$, Sutoh $\mathrm{T}$, Morita $\mathrm{H}$, et al. Serum albumin is superior to prealbumin for predicting short-term recurrence in patients with operable colorectal cancer. Nutr Cancer. 2012; 64(8): 1169-1173, doi: 10.1080/01635581.2012.718034, indexed in Pubmed: 23163845.

25. Håkonsen SJ, Pedersen PU, Bath-Hextall F, et al. Diagnostic test accuracy of nutritional tools used to identify undernutrition in patients with colorectal cancer: a systematic review. JBI Database System Rev Implement Rep. 2015; 13(4): 141-187, doi: 10.11124/jbisrir-2015-1673, indexed in Pubmed: 26447079

26. Tu MY, Chien TW, Chou MT. Using a nutritional screening tool to evaluate the nutritional status of patients with colorectal cancer. Nutr Cancer. 2012; 64(2): 323-330, doi: 10.1080/01635581.2012.650778, indexed in Pubmed: 22292458

27. van Bokhorst-de van der Schueren MAE, Guaitoli PR, Jansma EP, et al. Nutrition screening tools: does one size fit all? A systematic review of screening tools for the hospital setting. Clin Nutr. 2014; 33(1): 39-58, doi: 10.1016/j.clnu.2013.04.008, indexed in Pubmed: 23688831.

28. Rowan NR, Johnson JT, Fratangelo CE, et al. Utility of a perioperative nutritional intervention on postoperative outcomes in high-risk head \& neck cancer patients. Oral Oncol. 2016; 54: 42-46, doi: 10.1016/j. oraloncology.2016.01.006, indexed in Pubmed: 26803343.

29. Ell SR, Stell PM, Stell PM, et al. Prognostic factors in laryngeal carcinoma. Clin Otolaryngol Allied Sci. 1988; 13(5): 399-409, indexed in Pubmed: 3072131

30. Zemplén B, Döbrentey E, Ottó S, et al. [Survey of the nutritional status of patients with locally advanced laryngo-pharyngeal tumors]. Orv Hetil. 1989; 130(30): 1591-1595, indexed in Pubmed: 2505210. 